\title{
Molecular Modeling, Structural Analysis and Evaluation of 1-Deoxy-D-Xylulose-5-Phosphate Reductoisomerase (DXR) as a Putative Drug Target For Theileria Parva
}

\author{
Irmak Icen-Taskin ${ }^{1}$, Omer Munzuroglu ${ }^{2}$. \\ ${ }^{1}$ Firat University - Department of Biology, Faculty of Sciences, Elazig, Turkey; ${ }^{2}$ Department of Bioengineering, \\ Firat University, Elazig, Turkey.
}

\begin{abstract}
The apicomplexan parasite Theileria parva, the causative agent of ECF, is an important pathogen affecting both domestic and wild animals, causing major economic losses in the world. Problems such as high cost of drugs, development of resistance, and absence of effective vaccines prevent effective combating of the pathogen. Thus, it is necessary to explore new targets for affordable and higher therapeutic value drugs. 1-Deoxy-D-xylulose-5phosphate reductoisomerase $(D X R)$ in the non-mevalonate isoprene biosynthesis pathway is vital to the organism and therefore has been selected as a target for developing antitheilerial drugs. In this study, the $3 D$ structure of TpDXR was identified by template-based in silico homology modelling method, the constructed model was validated and structurally analysed, and possible ligand binding pockets were identified for the first time in the literature. A reliable 3D model for TpLDH was modelled by using $3 A U 9$ chain 'A' Plasmodium falciparum as a template. The obtained result showed that the model has a good resolution structure with 86.768 overall quality factor and a -9.15 $z$-score for TpDXR. The present study promises the possibility of exploiting new and safe inhibitors using the structure-based drug design that is effective against ECF through docking studies.
\end{abstract}

Key words: Homology modelling; Theileria parva; 1-Deoxy-D-xylulose-5-phosphate reductoisomerase (DXR); East Coast Fever

\footnotetext{
*Author for correspondence: irmak.icen@hotmail.com, iicen@firat.edu.tr
} 


\section{INTRODUCTION}

Theileria parva is one of the two economically important protozoa of the Theileria genus belonging to the apicomplexan phylum which includes Plasmodium ssp. and Toxoplasma gondii, causative agents of malaria and toxoplasmosis, respectively (1). Theileria parva is the disease agent of tick-borne East Coast Fever (ECF), which ranks first among the tick-borne diseases affecting cattle in sub-Saharan Africa (2). The disease caused by the parasite affects a large proportion of domestic and wild animals in the world. This parasite is highly pathogenic for cattle as the vertebrate host, leading to serious economic losses (3).

In general, the ECF therapy is based on controlling strategies that utilize acaricides for tick vector (4) immunization via the live attenuated cell-line vaccine (5) and the medical consensus that combines drugs such as parvaquone and buparvaquone $(6,7)$ for Theileria parva. On the other hand, the factors limiting the wide use of this treatment are the high costs of the acaricides that are utilized for tick control (8) and the rapid resistance development against a wide variety of these $(9,10)$, as well as the absence of an efficient vaccine against $T$. parva that is proven to be safe (4). Moreover, in 2010, resistance to buparvaquone has been observed as well (11); this was followed by a new report in the year 2012 caused by point mutation mitochondrial cytochrome $b$ gene in the parasite $(12,13)$. Considering these factors, the development of an efficient and affordable anti-theilerial agent in a different mode of action that can be used safely is vital.

With the complementation of the genome sequence of T.parva (14), improved knowledge about the biochemical pathways utilized by this organism has opened novel approaches for development of a chemotherapeutic agent. The 1-deoxy-Dxylulose 5-phosphate/2-C-methyl-D-erythritol-4- phosphate (DXP/MEP) pathway is one of the essential metabolic pathways targeted for rational drug discovery $(15,16)$. The MEP pathway is utilized by most bacteria and apicomplexan parasites, including Mycobacterium tuberculosis, Helicobacter pylori, T. gondii and Plasmodium spp. (malaria parasites), to synthesize isopentenyl diphosphate (IPP) and its isomer dimethylallyl diphosphate (DMAPP) for the synthesis of isoprenoid compounds. There is no functional equipollent of this pathway in mammals and since the DXP/MEP pathway is necessary in these organisms (15), the enzymes involved in this pathway become a promising target for novel therapeutic intervention, without harming host vertebrates. 1-Deoxy-D-xylulose-5-phosphate reductoisomerase (DXR) is the second enzyme of the pathway, catalysing the reduction and isomerization of 1-deoxy-D-xylulose-5-phosphate (DXP) to 2-Cmethyl-D-erythritol-4-phosphate (MEP) by utilizing Mg2+ and NADPH as enzyme cofactors. Potential inhibitor, fosmidomycin, a naturally occurring antibiotic against DXR, has already been identified and has demonstrated antimalarial activities in preclinical studies and clinical tribulations (17-19). These findings insinuate that DXR is a valid target, and inhibitors of this enzyme could serve as potential guides for apicomplexan's chemotherapy.

Even though DXR of Plasmodium spp. has been considered to identify a novel drug against malaria (17), unavailability of information about Thaileria parva 1-DeoxyD-xylulose-5-phosphate reductoisomerase (TpDXR) restricts rapid improvement of drug development against this protozoan pointed $T p D X R$. For this reason, a threedimensional model (3D) of $T p \mathrm{DXR}$ was created through comparative modelling for the first time ever, after intensive optimization, minimization, validation and identification of possible drug target pockets to open a new route to design specific inhibitors. 
A new drug target against ECF

\section{MATERIALS \& METHODS}

Template Selection, Physiochemical And Functional Properties And Secondary Structure Prediction

The amino acid sequence of the DXR from $T$. parva (TpDXR) (accession number XP_764642.1) was retrieved from the National Centre for Biotechnology Information database. The physiochemical features of $T p D X R$, such as theoretical isoelectric point (pI), molecular weight, the total number of positive and negative residues, extinction coefficient, instability index, aliphatic index and grand average hydropathy (GRAVY), were determined with the Expasy's ProtParam server (20). The secondary structure features of the $T p D X R$ were revealed with the web-based program SOPMA (21).

\section{Homology Model Development And Model Validation}

Initially, an automatic 3D model of TpDXR was constructed based on structural homologies with a template protein using the programme Protein Homology/analogy Recognition engine 2 (PHYRE2) (22), a free online homology modelling server. This program utilizes the alignment of hidden Markov models through HHsearch to remarkably increase the efficiency of alignment and recognition rate. Energy minimisation and root mean square deviation (RMSD) were performed using 3Drefine (23) and SuperPose servers (23), respectively. To ensure the quality of modelling, stereochemistry, energy profile and residue environment of modelled structure were checked by different servers. RAMPAGE (24) was used to test the stereochemistry of the model. The model was further examined by ProSA (25) and ERRAT servers (26). Protein Structure Validation Suite-PSVS 1.3 (http://psvs-1 4dev.nesg.org) was used to analyse the quality of the model.

\section{Ligand Binding Site Identification}

The MetaPocket 2.0 server (27) was used to distinguish ligand-binding sites on the protein surface. This server is employed as an agreement strategy to predicted binding sites from PASS11 (PAS), LigsiteCS (LCS), Q_SiteFinder (QSF), GHECOM (GHE), POCASA (PCS), Fpocket (FPK), SURFNET (SFN), and ConCavity (CON) methods.

\section{RESULTS AND DISCUSSION}

\section{Template Selection, Physiochemical And Functional Properties And Secondary} Structure Prediction

Theileria parva 1-deoxy-D-xylulose-5-phosphate reductoisomerase (TpDXR) (EC:1.1.1.267) has been selected in this study as a drug target, having previously been analysed extensively in other apicomplaxa $(28,29)$ but not in $T$. parva. $T p \mathrm{DXR}$ is one of the essential enzymes for the survival of the parasite, and therefore could be a putative drug target for the design and evaluation of a new class of antitheilerials. Expasy's ProtParam server was used to analyse the stereochemistry, energy profiles, potential errors, nonbonding interactions and some chemical and physical traits of $T p D X R$. 


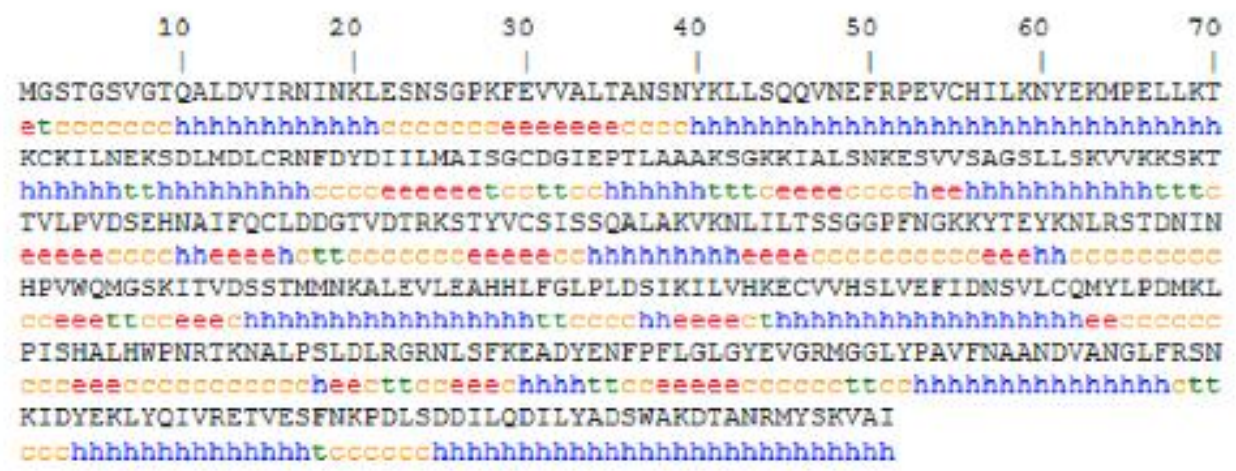

Fig. 1 : SOPMA server results for the secondary structure of the modelled TpDXR: Alpha helix (Hh), Extended strand (Ee), Beta-turn (Tt), Random coil (Cc)

The protein model was about $44617.2 \mathrm{kDa}$ and calculated $\mathrm{pI}$ (isoelectric point) of the TpDXP was $7.57 \quad(\mathrm{pI}>7)$. The total number of negatively charged residues (Asp + Glu) was \% 46 and \% 47 positively charged residues (Arg + Lys). The extinction coefficient of protein was 37,360 and the instability index of this enzyme was measured as 37.42 which indicated the protein was stable. The value for the aliphatic index was found to be 93.34. The grand average of hydropathicity (GRAVY) value was -0.216 . The secondary structure of $T p D X R$ was forecasted by the SOPMA server. According to the result, Alpha helix (46.88\%) and random coil (29.68\%) composed the large proportion of the physical feature. Extended strands and beta turns were determined to be $16.46 \%$ and $6.98 \%$, respectively (Fig. 1).

\section{Homology Model Development And Model Validation}

The template used for 3D structure modelling was 3AU9 chain 'A' Plasmodium falciparum (30), the resolution was $1.90 \AA$, which was analysed by X-ray diffraction and choosen in light of heuristics to expand certainty, percentage identity and alignment scope (Fig. 2).

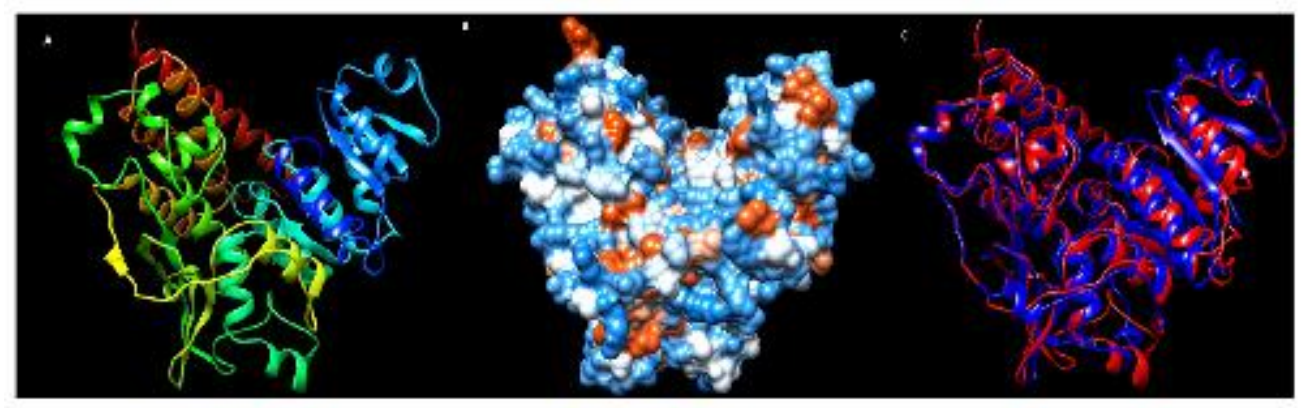

Fig. 2: Predicted 3D structure of TpDXR, A: Representation of TpDXR in ribbon style B: Surface representation of TpDXR . C Superposition of the predicted structureTpDXR with template (PDB access code: 3AU9)

The modelled structure refinement was achieved by local energy minimization and the final value was $-8102.2902 \mathrm{~kJ} / \mathrm{mol}$. The C $\alpha$ RMSD and the backbone RMSD deviations for the model and the template crystal structure were observed to be 0.85 and $0.86 \AA$, respectively. The Ramachandran plot for the anticipated model by RAMPAGE revealed that $95 \%$ (379) of residues were found in the most favourable region, $4 \%$ (16) of residues were in the allowed regions and $1 \%$ (4) of residues were in the outlier region (Fig. 3). 
A new drug target against ECF

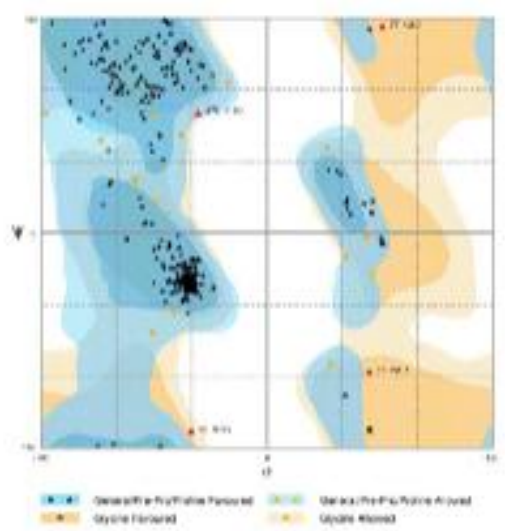

Fig. 3: Ramachandran plot of 1-deoxy-D-xylulose-5-phosphate Reductoisomerase from Theileria parva obtained by RAMPAGE: $95 \%$ residues in favourable regions; $4 \%$ residues in allowed regions; $1 \%$ residues in outlier region.

The ERRAT server was used to predict non-bonding interactions. The obtained result showed that the model had a good resolution structure with 86.768 overall quality factor (26). ProSA server was used for assessment of the overall quality of the modelled protein by means of the scores of all experimentally determined protein chains as of now accessible in the Protein Data Bank (PDB) (25). The program gave a $-9.15 \mathrm{z}$-score and a plot of the residue energies for $T p \mathrm{DXR}$ demonstrated that the modelled quality was acceptable (Fig. 4). The overall $G$ factor ascertained on the premise of the primary chain parameter construed that the modelled structure was satisfactory for virtual screening.
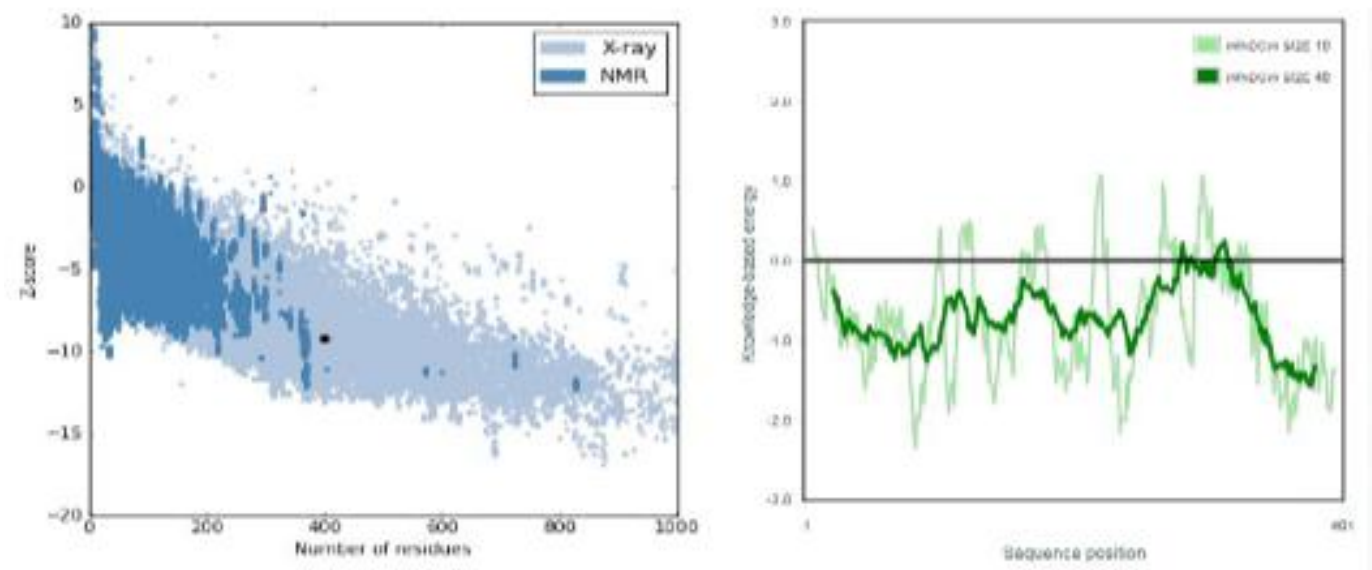

Fig. 4: ProSA web service analysis of $T p D X R$ protein model

\section{Ligand Binding Sites}

The potential binding sites (PBS) of proteins are those residues or atoms which tie to ligands directly on the protein surface. They are predicted by the MetaPocket 2.0 with distinctive strategies, such as PAS, QSF, FPK, SFN, GHE, CON, and LCS. We have considered the top three sites (Fig. 5). 

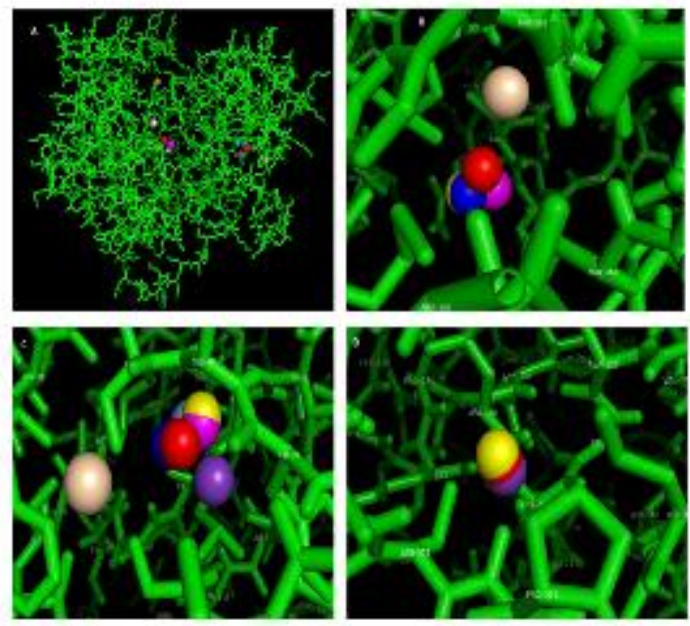

Fig. 5: The predicted potential top 3 binding sites in $T p D X R(A): M P K 1(B), \quad M P K 2(C)$, MPK3(D); Pocket color description are indicated as: red - MPK, actinium - PAS, magenta - QSF, potassium - FPK, wheat - SFN, yellow GHE, blue - CON and raspberry - PCS. The exact residue location information is given in Table 1.

After clustering the top three sites from PAS, LCS, FPK, SFN, GHE, and CON, six MetaPocket clusters were obtained. The initial MetaPocket site (MPK1) contained four pocket sites, namely, the first pocket from SURFNET(SFN-1), the first pocket from LigisiteCS (LCS-1), the first pocket from GHECOM (GHE-1), and the first pocket from Concavity (CON-1), with a total $19.16 \mathrm{z}$-score. The second MetaPocket site (MPK2) consisted of six pockets, namely, GHE-2, FPK-2 (Fpocket), LCS-2, SFN-2, PAS-2(PASS), and CON-2, with a total z- score of 11.19. The third MetaPocket site (MPK3) contained two pocket sites, namely, FPK-1 and GHE-3, with a total $4.18 \mathrm{z}$-score. The fourth MetaPocket site contained four pocket sites, namely, SFN-3, LCS-3, CON-3 and PAS-1, with a total 3.95 z-score. The fifth MetaPocket site contained one pocket site, FPK-3, with a total $1.66 \mathrm{z}$-score. The last MetaPocket site contained one pocket site, PAS-3, with a total z-score of 0.55. Table 1 shows the potential binding sites from the modelled $T p D X R$ residue.

Table 1 : Identified possible ligand binding pockets:

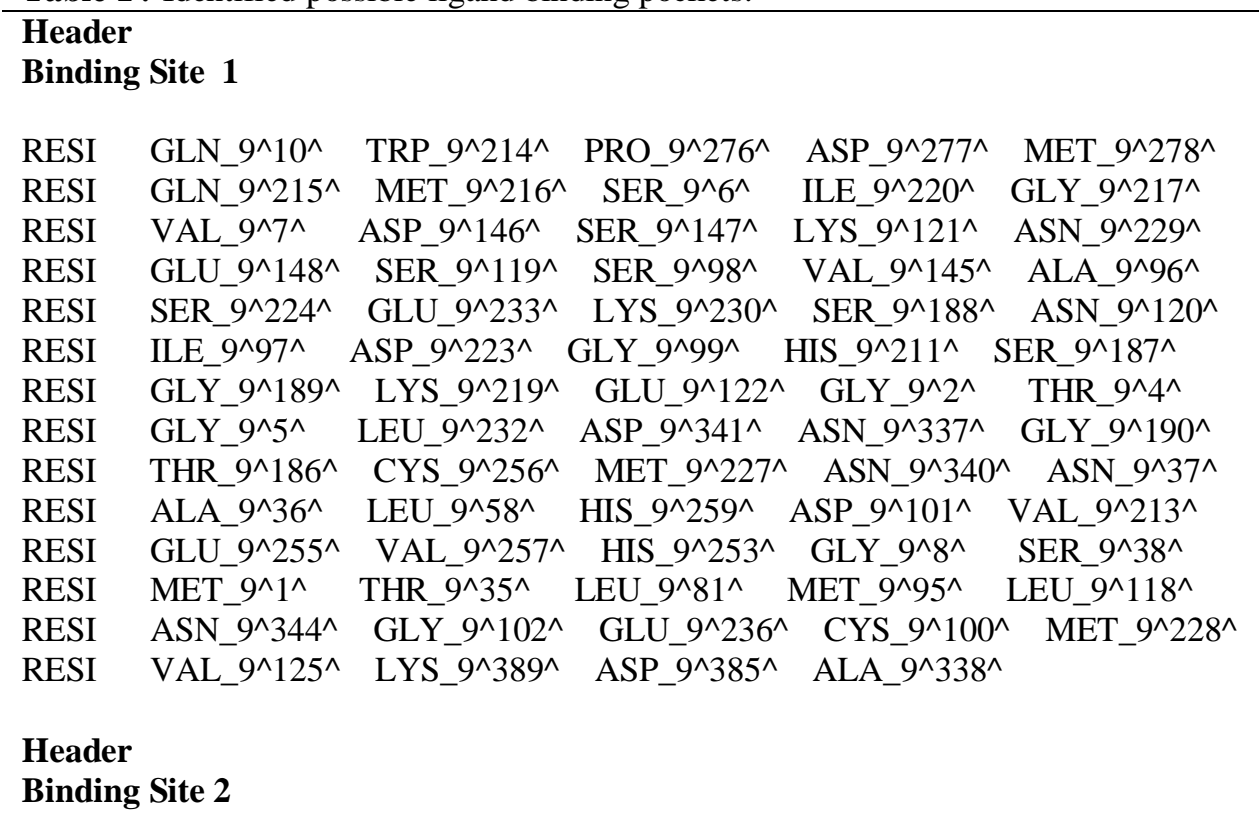


A new drug target against ECF

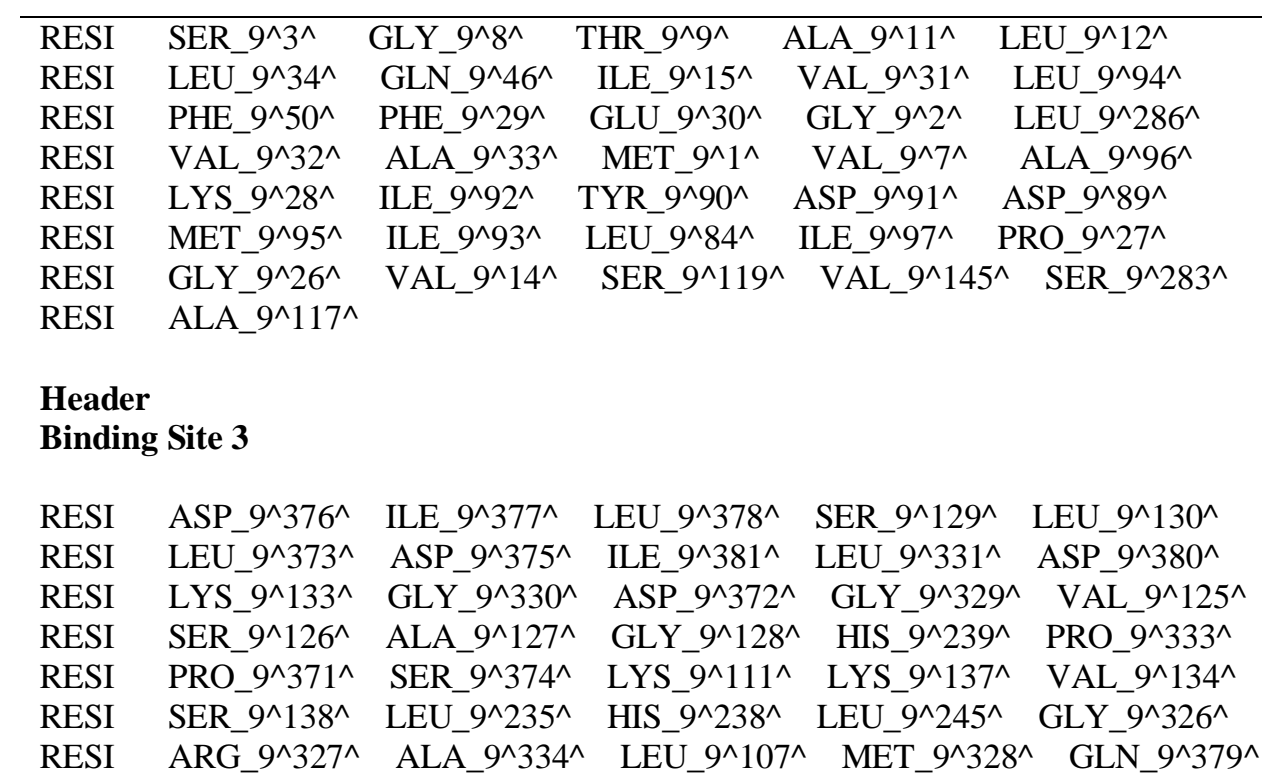

The potential binding sites of three MetaPockets are shown and they are present in residue format with each line starting with 'RESI'. The residue specificated in the table is constructed of three parts: residue name, chain indicator and residue sequence number.

\section{CONCLUSIONS}

The DXR has been proposed as a drug target (e.g. fosmidomycin, Fos) against apicomplexan parasites (17). Fosmidomycin is used widely as a DXR inhibitor. In a clinical study, fosmidomycin-clindamycin was found to be efficient in the treatment of 105 Gabonese patients representing children aged 3 to 14 years with uncomplicated malaria, demonstrating its application in clinical trials (19). Moreover, eleven mixes with expansive basic assorted qualities were tried against Toxoplasma gondii DXR and a few strong inhibitors were identified with very low $48 \mathrm{nM} \mathrm{Ki}$ values (29). These outcomes show DXR is an important target, and conceivable inhibitors of this compound may serve as a potential route for ECF chemotherapy. However, even though significant data were obtained about the metabolism of the parasite through the complementation genome project of T.parva in 2005, no additional study about $T p D X R$ was conducted. In the present work, a computational molecular modelling method was applied to resolve the threedimensional structure of $T p D X R$, to overcome the lack of its crystallographic structure. After a few stages of energy minimization and refinement, possible binding pockets were identified. These results have critical ramifications for future screens aimed to discover new and safe molecular entities with activity against TpDXR.

\section{REFERENCES}

1. Shaw MK. The same but different: the biology of Theileria sporozoite entry into bovine cells. Int J Parasitol. 1997;27(5):457-74.

2. Bruno Minjauw AM. Tick-borne diseases and poverty: the impact of ticks and tick-borne diseases on the livelihoods of small-scale and marginal livestock owners in India and eastern and southern Africa. Research report. 2003.

3. Mukhebi AW, Perry BD, Kruska R. Estimated Economics of Theileriosis Control in Africa. Prev Vet Med. 1992;12(1-2):73-85. 
4. Walker AR. Theileriosis and the tick control conundrum: A better way forward? Vet J. 2007;173(2):248-9.

5. Bishop R, Geysen D, Spooner P, Skilton R, Nene V, Dolan T, et al. Molecular and immunological characterisation of Theileria parva stocks which are components of the 'Muguga cocktail' used for vaccination against East Coast fever in cattle. Vet Parasitol. 2001;94(4):227-37.

6. Dolan TT, Injairu R, Gisemba F, Maina JN, Mbadi G, Mbwiria SK, et al. A clinical trial of buparvaquone in the treatment of East Coast fever. Vet Rec. 1992;130(24):536-8.

7. McHardy N, Wekesa LS, Hudson AT, Randall AW. Antitheilerial activity of BW720C (buparvaquone): a comparison with parvaquone. Res Vet Sci. 1985;39(1):29-33.

8. Walker AR. Theileriosis and the tick control conundrum: a better way forward? Vet J. 2007;173(2):248-9.

9. Benavides E, Rodriguez JL, Romero A. Isolation and partial characterization of the Montecitos strain of Boophilus microplus (Canestrini, 1877) multiresistant to different acaricides. Ann N Y Acad Sci. 2000;916:668-71.

10. Miller RJ, Davey RB, George JE. Modification of the food and agriculture organization larval packet test to measure amitraz-susceptibility against ixodidae. J Med Entomol. 2002;39(4):645-51.

11. Mhadhbi M, Naouach A, Boumiza A, Chaabani MF, BenAbderazzak S, Darghouth MA. In vivo evidence for the resistance of Theileria annulata to buparvaquone. Vet Parasitol. 2010;169(3-4):241-7.

12. Sharifiyazdi H, Namazi F, Oryan A, Shahriari R, Razavi M. Point mutations in the Theileria annulata cytochrome $\mathrm{b}$ gene is associated with buparvaquone treatment failure. Vet Parasitol. 2012;187(3-4):431-5.

13. Mhadhbi M, Chaouch M, Ajroud K, Darghouth MA, BenAbderrazak S. Sequence Polymorphism of Cytochrome $b$ Gene in Theileria annulata Tunisian Isolates and Its Association with Buparvaquone Treatment Failure. PLoS One. 2015;10(6):e0129678.

14. Gardner MJ, Bishop R, Shah T, de Villiers EP, Carlton JM, Hall N, et al. Genome sequence of Theileria parva, a bovine pathogen that transforms lymphocytes. Science. 2005;309(5731):134-7.

15. Singh N, Cheve G, Avery MA, McCurdy CR. Targeting the methyl erythritol phosphate (MEP) pathway for novel antimalarial, antibacterial and herbicidal drug discovery: inhibition of 1-deoxy-D-xylulose-5-phosphate reductoisomerase (DXR) enzyme. Curr Pharm Des. 2007;13(11):1161-77.

16. Obiol-Pardo C, Rubio-Martinez J, Imperial S. The Methylerythritol Phosphate (MEP) Pathway for Isoprenoid Biosynthesis as a Target for the Development of New Drugs Against Tuberculosis. Curr Med Chem. 2011;18(9):1325-38.

17. Jomaa H, Wiesner J, Sanderbrand S, Altincicek B, Weidemeyer C, Hintz M, et al. Inhibitors of the nonmevalonate pathway of isoprenoid biosynthesis as antimalarial drugs. Science. 1999;285(5433):1573-6.

18. Missinou MA, Borrmann S, Schindler A, Issifou S, Adegnika AA, Matsiegui PB, et al. Fosmidomycin for malaria. Lancet. 2002;360(9349):1941-2.

19. Oyakhirome S, Issifou S, Pongratz P, Barondi F, Ramharter M, Kun JF, et al. Randomized controlled trial of fosmidomycin-clindamycin versus sulfadoxinepyrimethamine in the treatment of Plasmodium falciparum malaria. Antimicrob Agents Chemother. 2007;51(5):1869-71.

20. Gasteiger E, Gattiker A, Hoogland C, Ivanyi I, Appel RD, Bairoch A. ExPASy: The proteomics server for in-depth protein knowledge and analysis. Nucleic Acids Res. 2003;31(13):3784-8.

21. Geourjon C, Deleage G. SOPMA: significant improvements in protein secondary structure prediction by consensus prediction from multiple alignments. Comput Appl Biosci. 1995;11(6):681-4.

22. Kelley LA, Mezulis S, Yates CM, Wass MN, Sternberg MJ. The Phyre2 web portal for protein modeling, prediction and analysis. Nat Protoc. 2015;10(6):845-58.

23. Bhattacharya D, Cheng J. 3Drefine: consistent protein structure refinement by optimizing hydrogen bonding network and atomic-level energy minimization. Proteins. 2013;81(1):119-31. 
A new drug target against ECF

24. Lovell SC, Davis IW, Arendall WB, 3rd, de Bakker PI, Word JM, Prisant MG, et al. Structure validation by Calpha geometry: phi,psi and Cbeta deviation. Proteins. 2003;50(3):437-50.

25. Wiederstein M, Sippl MJ. ProSA-web: interactive web service for the recognition of errors in three-dimensional structures of proteins. Nucleic Acids Res. 2007;35(Web Server issue):W407-10.

26. Colovos C, Yeates TO. Verification of protein structures: patterns of nonbonded atomic interactions. Protein Sci. 1993;2(9):1511-9.

27. Huang B. MetaPocket: a meta approach to improve protein ligand binding site prediction. OMICS. 2009;13(4):325-30.

28. Odom AR, Van Voorhis WC. Functional genetic analysis of the Plasmodium falciparum deoxyxylulose 5-phosphate reductoisomerase gene. Mol Biochem Parasitol. 2010;170(2):108-11.

29. Cai G, Deng L, Xue J, Moreno SN, Striepen B, Song Y. Expression, characterization and inhibition of Toxoplasma gondii 1-deoxy-D-xylulose-5-phosphate reductoisomerase. Bioorg Med Chem Lett. 2013;23(7):2158-61.

30. Umeda T, Tanaka N, Kusakabe Y, Nakanishi M, Kitade Y, Nakamura KT. Molecular basis of fosmidomycin's action on the human malaria parasite Plasmodium falciparum. Sci Rep. 2011;1:9.

Received: February 03, 2016; Accepted: July 14, 2016 